\title{
Future projects of light kaonic atom X-ray spectroscopy
}

\author{
H. Tatsuno ${ }^{1, \star}$, M. Bazzi ${ }^{2}$, G. Beer ${ }^{3}$, G. Bellotti ${ }^{4,5}$, C. Berucci ${ }^{2,6}$, A.M. Bragadireanu 2,7 , D. Bosnar ${ }^{8}$,
} M. Cargnelli, , C. Curceanu' ${ }^{2}$, A.D. Butt ${ }^{4,5}$, A. d'Uffizi ${ }^{2}$, C. Fiorini ${ }^{4,5}$, F. Ghio ${ }^{9}$, C. Guaraldo ${ }^{2}$, R.S. Hayano ${ }^{10}$, M. Iliescu ${ }^{2}$, T. Ishiwatari ${ }^{6}$, M. Iwasaki ${ }^{11}$, P. Levi Sandri' ${ }^{2}$, J. Marton ${ }^{6}$, S. Okada ${ }^{12}$, D. Pietreanu 2,7 , K. Piscicchiaa,13, A. Romero Vidal ${ }^{14}$, E. Sbardella ${ }^{2}$, A. Scordo ${ }^{2}$, H. Shi ${ }^{2}$, D.L. Sirghi ${ }^{2,7}$, F. Sirghi ${ }^{2,7}$, O. Vazquez Doce ${ }^{15}$, E. Widmann ${ }^{6}$, and J. Zmeskal ${ }^{6}$ for the SIDDHARTA Collaboration

${ }^{1}$ Department of Chemical Physics, Lund University, Lund, Sweden

2 INFN, Laboratori Nazionali di Frascati, Frascati (Roma), Italy

${ }^{3}$ Department of Physics and Astronomy, University of Victoria, Victoria BC, Canada

${ }^{4}$ Politecnico di Milano, Dipartimento di Elettronica e Informazione, Milano, Italy

${ }^{5}$ INFN Sezione di Milano, Milano, Italy

${ }^{6}$ Stefan-Meyer-Institut für subatomare Physik, Wien, Austria

${ }^{7}$ IFIN-HH, Institutul National pentru Fizica si Inginerie Nucleara Horia Hulubbei, Magurele, Romania

${ }^{8}$ Department of Physics, Faculty of Science, University of Zagreb, Zagreb, Croatia

9 INFN Sezione di Roma I and Istituto Superiore di Sanità, Roma, Italy

${ }^{10}$ Department of Physics, School of Science, The University of Tokyo, Tokyo, Japan

${ }^{11}$ RIKEN Nishina Center, RIKEN, Wako, Japan

${ }^{12}$ AMO physics laboratory, RIKEN, Wako, Japan

${ }^{13}$ Museo Storico della Fisca e Centro Studi e Ricerche "Enrico Fermi", Roma, Italy

${ }^{14}$ Universidade de Santiago de Compostela, Santiago de Compostela, Spain

${ }^{15}$ Excellence Cluster Universe, Technische Universität München, Garching, Germany

\begin{abstract}
X-ray spectroscopy of light kaonic atoms is a unique tool to provide precise information on the fundamental $\bar{K} N$ interaction at the low-energy limit and the in-medium nuclear interaction of $K^{-}$. The future experiments of kaonic deuterium strong-interaction shift and width (SIDDHARTA-2 and J-PARC E57) can extract the isospin dependent $K^{-} N$ interaction at threshold. The high-resolution X-ray spectroscopy of kaonic helium with microcalorimeters (J-PARC E62) has the possibility to solve the long-standing potential-strength problem of the attractive $K^{-}$-nucleus interaction. Here, the recent experimental results and the future projects of X-ray spectroscopy of light kaonic atoms are presented.
\end{abstract}

\section{Introduction}

The kaon is one of the pseudo-scalar mesons, the mass (495 MeV) lies between that of pion (140 $\mathrm{MeV})$ and eta $(510 \mathrm{MeV})$ mesons, and the anti-kaon $\bar{K}\left(\bar{K}^{0}\right.$ and $\left.K^{-}\right)$includes a strange quark $(\bar{d} s$ and $\bar{u} s$ ). Therefore the anti-kaon is a unique and suitable particle to study the role of explicit chiral

^e-mail: hideyuki.tatsuno@gmail.com, 
symmetry breaking due to the relatively heavier strange quark and the dynamics of low-energy QCD in the strangeness sector. The in-medium nuclear interaction of the $K^{-}$is also important to understand the structure of exotic nuclear states such as the $\bar{K} N$ sub-threshold resonance $\Lambda(1405)$, the nuclear bound state of $K^{-}$, as well as the high density of matter in the universe (neutron stars).

The precise study of the $\bar{K} N$ strong interaction has started from X-ray spectroscopy of kaonic atoms. The kaonic atom is a Coulomb-bound system of a $K^{-}$, electrons, and a nucleus. The transition X-ray energy is typically several $\mathrm{keV}$ for light kaonic atoms due to the $K^{-} /$electron mass ratio of $\sim 1000$. The $\bar{K} N$ interaction is attractive at short range, thus the strong interaction effects appear on the atomic states as the perturbation; an energy shift from the QCD calculation and a life-time broadening due to nuclear absorption of $K^{-}$. The $1 s$-state strong-interaction shifts and widths of kaonic hydrogen $\left(K^{-}-p\right)$ and kaonic deuterium $\left(K^{-}-d\right)$ can be used to directly extract the isospin dependent $K^{-} N$ interaction at threshold by the Deser-Trueman type formula [1]. They are necessary for precise calculations of chiral SU(3) meson-baryon effective-field theory in low-energy QCD. Furthermore, the $K^{-} N$ threshold information provides the basic constraint on the structure of the $\Lambda(1405)[2,3]$. The X-ray spectroscopy of other kaonic atoms (helium to uranium) enables the study of the strong interaction of the $K^{-}$in dense nuclei. The bound state of a $K^{-}$in a nucleus has been predicted using the strongly attractive interaction [4], and recently the $K^{-}$multi-nucleon cluster system like $K^{-} p p$ is one of the hot topics in this field.

Currently, the strong-interaction shifts and widths of kaonic atoms from hydrogen to uranium have been measured. The $K^{-} p$ scattering length has been determined, and also the $K^{-}$-nucleus interaction is qualitatively understood. A systematic analysis with a phenomenological density-dependent optical potential can accurately describe the trend of shift and width of kaonic atoms and predict the deep potential depth $\left(\operatorname{Re}\left(V_{0}\right) \sim-180 \mathrm{MeV}\right)[5,6]$. In addition to that, a chiral $\mathrm{SU}(3)$ calculation which is based on the experimental data of the $\bar{K} N$ interaction can also describe the experimental data of shifts and widths and predict the relatively shallow potential depth $\left(\operatorname{Re}\left(V_{0}\right) \sim-50 \mathrm{MeV}\right)$ [7]. This quantitative disagreement, so-called 'deep-or-shallow problem' is fundamentally correlated to the characteristic and structure of the $K^{-}$multi-nucleon cluster states. Hence, a more precise and decisive experimental measurement has been awaited.

We present here the key experiments and the future projects of light kaonic atom X-ray spectroscopy:

1. past and recent results of kaonic hydrogen $2 p \rightarrow 1 s$ X-ray measurement by KEK-PS E228 [8], DEAR [9], and SIDDHARTA [10],

2. first measurement of kaonic deuterium $2 p \rightarrow 1 s$ X-rays planned by the SIDDHARTA-2 [11] and the J-PARC E57 [12] groups,

3. high-resolution x-ray spectroscopy of kaonic helium-3 and helium-4 with superconducting transition-edge-sensors (TESs) by the HEATES and J-PARC E62 groups [13], which can solve the deep-or-shallow problem on the $K^{-}$-nucleus potential depth.

\section{Kaonic hydrogen}

The primary study of the $\bar{K} N$ interaction based on the $K^{-} p$ scattering experiments in the 1960s-1980s. The threshold information was extrapolated with the cross section data with large uncertainties. At that time, the precise measurement technique of kaonic hydrogen X-rays was not yet established; therefore even the sign of the $\bar{K} N$ interaction (attractive or repulsive) was not determined. Later, the 
kaonic hydrogen X-ray measurement with new background suppression technique and with new Xray detectors was performed by three experimental groups. The details of experiments are shown in Table 1.

The reliable $\bar{K} N$ threshold information was first provided in the 1990s. The KEK-PS E228 group measured the strong-interaction shift and width of $K^{-}-p 1 s$ state at KEK-PS in Tsukuba, Japan [8]. The $2 p \rightarrow 1 s$ X-ray energy is about $6.2 \mathrm{keV}$, and the strong interaction shift is $\sim-300 \mathrm{eV}$ (repulsive) and the width is $\sim 400 \mathrm{eV}$. The $K^{-}$beam extracted through the $\mathrm{K} 3$ secondary beam line was slowed down with carbon degraders and stopped on the hydrogen gaseous target. They measured the X-rays with $\mathrm{Si}(\mathrm{Li})$ detectors installed inside of the target chamber and also tracked the coincident secondary charged particles generated from the reaction of $K^{-}$and nucleus with a cylindrical drift chamber system. The event selection of specific reaction modes and the timing selection of stopped $K^{-}$successfully reduced the synchronous background, and they clearly observed the clear peaks of $K^{-}$- $p$ X-rays. Though the background was small, the statistical uncertainties of the shift and width were large (see Table 1), because the energy resolution of the $\mathrm{Si}(\mathrm{Li})$ detectors was $360 \mathrm{eV}$ full-widthat-half-maximum (FWHM) at $6 \mathrm{keV}$ (this is relatively worse than recent silicon detectors of $150 \mathrm{eV}$ FWHM). However, it was enough to determine the sign of the $\bar{K} N$ interaction (attractive) and laid the basis of theory. The attractive $\bar{K} N$ interaction led to the prediction of the deeply-bound $K^{-}$nuclear states [4]. The established event selection technique at the high intensity hadron beam line has been transitioned to other kaonic atom measurements at KEK and J-PARC in Tokai, Japan $[12,14]$.

In 2005, the DEAR group measured the strong-interaction shift and width of kaonic hydrogen at the DAФNE positron-electron collider in Frascati, Italy [9]. DAФNE produced almost at rest $\phi(1020)$ mesons with $510 \mathrm{MeV} e^{+}$and $e^{-}$beams. The low kinetic energy $K^{-} \mathrm{s}$ from $\phi \rightarrow K^{+} K^{-}$decay were easily stopped in the gaseous target. They measured the $K^{-}-p \mathrm{X}$-rays with the CCD detectors with $180 \mathrm{eV}$ FWHM energy resolution at $6 \mathrm{keV}$. Although the accumulated number of events was high, the background was also large due to no timing information of the CCDs. The background level of a lepton collider is usually lower than that of a hadron beam line. However, the signal-to-background ratio depends on the yield of the interesting event, and in the DEAR case, the $K^{-}-p \mathrm{X}$-ray yield is $\sim 1 \%$ per stopped kaon in the $24 \rho_{\text {STP }}$ density hydrogen target $\left(\rho_{\text {STP }}\right.$ is the density at the standard temperature and pressure). They subtracted the large background and characteristic X-ray peaks of ion and manganese which were overlaid with the $K^{-}-p \mathrm{X}$-rays, and then determined the shift and width. After the DEAR result was published, the KEK-PS E228 and DEAR results suffered from theoretical ambiguities even with considering their experimental uncertainties [15].

The most precise and new results were provided by the SIDDHARTA group in 2011 at DAФNE. They used newly developed silicon drift detectors (SDDs) with $150 \mathrm{eV}$ FWHM energy resolution at $6 \mathrm{keV}$. They detected the decay charged kaons and created the hardware 'kaon' trigger. The trigger system and the sub-micro second timing resolution of SDDs successfully worked to reduce the background, and the significantly clear $K^{-}-p \mathrm{X}$-ray peaks were observed. One of the most remarkable things was the global analysis with $K^{-}-p$ and $K^{-}-d \mathrm{X}$-ray spectra. The measured X-ray spectrum with the deuterium target was useful to confirm the background shape and X-ray peaks of other kaonic atoms $\left(K^{-}-\mathrm{C}, K^{-}-\mathrm{N}, K^{-}-\mathrm{O}\right.$, and $\left.K^{-}-\mathrm{Al}\right)$, because the $K^{-}-d$ X-ray yield was under the background level. The precise $K^{-} p$ scattering length of the SIDDHARTA results provided new basic constrains for the $\bar{K} N$ interaction of chiral approach calculations (e.g., [3]).

\section{Kaonic deuterium}

The SIDDHARTA results of kaonic hydrogen give the strong constraint for the $K^{-} p$ scattering length at the threshold. On the other hand, the calculation of $K^{-} n$ scattering length (pure isospin=1 term) is 
Table 1. Experimental details of kaonic hydrogen X-ray spectroscopy $[8-10,16]$, where $\rho_{\text {STP }}$ is the density at the standard temperature and pressure, $\Delta E_{\mathrm{FWHM}}$ is the full-width-at-half-maximum energy resolution, and $\Delta t_{\text {FWHM }}$ is the time resolution. The first error of shift and width is statistical and second one is systematic.

\begin{tabular}{lccc}
\hline & KEK-PS E228 (1997) & DEAR (2005) & SIDDHARTA (2011) \\
\hline Place & KEK-PS K3 (Japan) & DAФNE (Italy) & DAФNE (Italy) \\
$\mathrm{H}_{2}$ gas density & $10 \rho_{\text {STP }}$ & $24 \rho_{\text {STP }}$ & $15 \rho_{\text {STP }}$ \\
X-ray detector & $\mathrm{Si}(\mathrm{Li}) 120 \mathrm{~cm}^{2}$ & $\mathrm{CCD} 116 \mathrm{~cm}^{2}$ & SDD $114 \mathrm{~cm}^{2}$ \\
$\Delta E_{\text {FWHM }}$ at $6 \mathrm{keV}$ & $360 \mathrm{eV}$ (beam off) & $180 \mathrm{eV}$ & $150 \mathrm{eV}$ \\
$\Delta t$ FWHM & $290 \mathrm{~ns}$ & - & $800 \mathrm{~ns}$ \\
$1 s$ shift $(\mathrm{eV})$ & $-323 \pm 63 \pm 11$ & $-193 \pm 37 \pm 6$ & $-283 \pm 36 \pm 6$ \\
$1 s$ width $(\mathrm{eV})$ & $407 \pm 208 \pm 100$ & $249 \pm 111 \pm 30$ & $541 \pm 89 \pm 22$ \\
$2 p \rightarrow 1 s$ X-ray yield & $1.5 \pm 0.5 \% /$ stopped $K^{-}$ & - & $1.2_{-0.3}^{+0.4} \% /$ stopped $K^{-}$ \\
\hline
\end{tabular}

totally varied for theories [3]. To determine the isospin=1 term of the scattering length, the essential measurement of kaonic deuterium $1 s$-state strong-interaction shift and width has been awaited for a long time. There is no evidence of the $K^{-}-d \mathrm{X}$-rays so far due to the low X-ray yield $(\sim 10 \%$ of kaonic hydrogen) and the broad width $(650-1000 \mathrm{eV}$ predicted by theory) $[17,18]$. In order to measure such a low-yield and broad X-ray peaks, a high-efficient and low-background measurement is required. Aimed at the first observation of the $K^{-}-d \mathrm{X}$-rays, two experiments are planned at DAФNE and J-PARC. Table 2 shows the expected parameters of the future experiments.

The SIDDHARTA-2 group plans to measure the $K^{-}-d$ X-rays from a gaseous deuterium target at DAФNE [11]. They improve the event selection efficiency and the background suppression. The efficiency of event selection is improved by a $K^{+}$-tag counter to identify the $K^{-}$from $\phi \rightarrow K^{+} K^{-}$decay. The synchronous background is suppressed by VETO counters to catch the coincident secondary charged particles generated from the $K^{-}$-nucleus reactions. They are also developing new SDDs (total $200 \mathrm{~cm}^{2}$ ) with $130 \mathrm{eV} \mathrm{FWHM} \mathrm{energy} \mathrm{resolution} \mathrm{at} 6 \mathrm{keV}$ and $400 \mathrm{~ns}$ timing resolution. Finally, these updates will achieve a 10-times higher signal-to-background ratio than the SIDDHARTA measurement.

The J-PARC E57 experiment will measure the $K^{-}-d \mathrm{X}$-rays from a gaseous deuterium target at J-PARC [12]. They use the high-intensity $K^{-}$beam at the K1.8 BR beam line. The $K^{-}-d \mathrm{X}$-rays are detected with new SDDs (total $246 \mathrm{~cm}^{2}$ ) with conjugation of a cylindrical drift chamber system. The background suppression by the event selections of stopped- $K^{-}$and the $K^{-} d$ reaction modes will result in a comparable signal-to-background ratio of SIDDHARTA-2.

\section{Kaonic helium}

Helium is a nucleus and its nuclear density can be treated as a medium for the $K^{-}$. The kaonic helium $\left(K^{-}{ }^{4} \mathrm{He}\right.$ and $\left.K^{-}{ }_{-}^{3} \mathrm{He}\right)$ is one of the good probes to investigate the medium effect of the $K^{-}$and the exotic $K^{-}$-bound states in nuclei. While the direct information of the $K^{-}$-nucleus interaction could be extracted from the strong-interaction $1 s$-state shift and width of kaonic helium, the $2 p \rightarrow 1 s$ X-ray has not been observed due to the low X-ray yield. This indicates that the $K^{-}$is most likely absorbed before the transition to the $1 s$ state. Hence, the observable strong-interaction shift and width of kaonic helium are on the $2 p$ state.

The kaonic helium $2 p$-state strong-interaction shift is predicted to be $\sim 0 \mathrm{eV}$, and the width to be a few $\mathrm{eV}$ by both the phenomenological 'deep' optical potential model $[5,6]$ and the chiral unitary 'shallow' potential model [7]. Recently, a possibility to solve the deep-or-shallow problem has been 
Table 2. Expected experimental details of kaonic deuterium X-ray spectroscopy [11, 12].

\begin{tabular}{lcc}
\hline & SIDDHARTA-2 (DAФNE) & J-PARC E57 (K1.8 BR) \\
\hline $\mathrm{D}_{2}$ gas density & $3 \%$ of liquid $\mathrm{D}_{2}$ & $5 \%$ of liquid $\mathrm{D}_{2}$ \\
X-ray yield & $0.1 \% /$ stopped $K^{-}$ & $0.1 \% /$ stopped $K^{-}$ \\
X-ray detector & SDD $200 \mathrm{~cm}^{2}$ & SDD $246 \mathrm{~cm}^{2}$ \\
$\Delta E_{\text {FWHM }}$ at $6 \mathrm{keV}$ & $130 \mathrm{eV}$ & $130 \mathrm{eV}$ \\
$\Delta t$ FWHM & $400 \mathrm{~ns}$ & $400 \mathrm{~ns}$ \\
Signal/BG & $1 / 3$ & $1 / 3$ \\
Precision $1 s$ shift & $\pm 30 \mathrm{eV}$ & $\pm 60 \mathrm{eV}$ \\
Precision $1 s$ width & $\pm 70 \mathrm{eV}$ & $\pm 130 \mathrm{eV}$ \\
Assumed $1 s$ width & $750 \mathrm{eV}$ & $800 \mathrm{eV}$ \\
Feature & high geometrical efficiency, & high beam intensity, event selection \\
& new trigger and VETO counters & with cylindrical drift chamber system \\
\hline
\end{tabular}

Table 3. Experimental results and details of kaonic helium X-ray spectroscopy [14, 19-22]. The first error of shift and width is statistical and second one is systematic.

\begin{tabular}{lccc}
\hline & KEK-PS E570 (2007) & SIDDHARTA (2011) & J-PARC E62 \\
\hline Place & KEK-PS K5 & DAФNE & J-PARC K1.8 BR \\
Target & liquid ${ }^{4} \mathrm{He}$ & ${ }^{4} \mathrm{He}$ gas $/{ }^{3} \mathrm{He}$ gas & liquid ${ }^{4} \mathrm{He} /$ liquid ${ }^{3} \mathrm{He}$ \\
X-ray detector & SDD $8 \mathrm{~cm}^{2}$ & SDD $144 \mathrm{~cm}^{2}$ & 240-pixel TES $23 \mathrm{~mm}^{2}$ \\
$\Delta E_{\text {FWHM }}$ at $6 \mathrm{keV}$ & $190 \mathrm{eV}$ & $150 \mathrm{eV}$ & $5 \mathrm{eV}$ \\
$\Delta t_{\text {FWHM }}$ & $380 \mathrm{~ns}$ & $800 \mathrm{~ns}$ & $1 \mu \mathrm{s}$ \\
$2 p$ shift & $+2 \pm 2 \pm 2 \mathrm{eV}\left({ }^{4} \mathrm{He}\right)$ & $+5 \pm 3 \pm 4 \mathrm{eV}\left({ }^{4} \mathrm{He}\right)$ & goal $\pm 0.2 \mathrm{eV}\left({ }^{4} \mathrm{He}-{ }^{3} \mathrm{He}\right)$ \\
& & $-2 \pm 2 \pm 4 \mathrm{eV}\left({ }^{3} \mathrm{He}\right)$ & \\
$2 p$ width & $\leq 18 \mathrm{eV}(90 \% \mathrm{CL})$ & $14 \pm 8 \pm 5 \mathrm{eV}\left({ }^{4} \mathrm{He}\right)$ & sensitive to determine \\
& & $6 \pm 6 \pm 7 \mathrm{eV}\left({ }^{3} \mathrm{He}\right)$ & \\
\hline
\end{tabular}

discussed with new theoretical calculations [23]. The isotope shift of $K^{-}{ }^{4} \mathrm{He}$ and $K^{-}{ }^{3} \mathrm{He} 2 p$ states (the difference of two shifts) is calculated to be $0.6 \mathrm{eV}$ for the deep potential [24] and $0.0 \mathrm{eV}$ for the shallow one [25]. Therefore, the two models can be distinguished experimentally with the precision of $0.2 \mathrm{eV}$. On the other hand, the current best precision of the $2 p$-state shift is $\pm 2 \mathrm{eV}$ (stat.) $\pm 2 \mathrm{eV}$ (syst.) [14]. At least one order of magnitude higher precision is necessary to distinguish the predictions. In order to achieve such a high precision, new measurement technique and low noise $\mathrm{X}$-ray detectors are needed.

The HEATES group has attempted to apply the superconducting TES microcalorimeters to highresolution hadronic atom X-ray spectroscopy. The TES is an ideal detector to measure a thin X-ray line like kaonic helium $3 d \rightarrow 2 p \mathrm{X}$-ray and to determine the X-ray energy precisely. According to the results of feasibility test experiment [26], the 240-pixel TES array (23 $\mathrm{mm}^{2}$ effective area) has achieved $5 \mathrm{eV}$ FWHM energy resolution at $6 \mathrm{keV}$ with $1 \mu$ s time resolution, and they successfully measured the pionic carbon $4 f \rightarrow 3 d \mathrm{X}$-ray energy with precision of $\leq 0.2 \mathrm{eV}$ [27].

The J-PARC E62 group plans the measurement of both the $K^{-}{ }_{-}^{4} \mathrm{He}$ and $K^{-}{ }_{-}^{3} \mathrm{He} \mathrm{X}$-rays with the TESs at the J-PARC K1.8 BR beam line [13]. The successive measurement can minimize the systematic uncertainty of isotope shift, and it is free from the theoretical uncertainty associated with the error of charged kaon mass. The recent results and the future experiment of kaonic helium X-ray spectroscopy are summarized in Table 3. 


\section{Summary}

The experimental results and the future projects of light kaonic atom spectroscopy are presented with the details of experimental methods.

The precise measurement of kaonic hydrogen X-rays by the SIDDHARTA group provided a unique possibility to determine the $K^{-} p$ scattering lengths. This is one of the most important observables to investigate the chiral SU(3) dynamics in low-energy QCD. For further study of the isospin dependence of the $K^{-} N$ interaction, the SIDDHARTA-2 and the J-PARC E57 groups plan the kaonic deuterium X-ray measurement with new SDDs. These measurements will provide constraints on the structure of the $\Lambda(1405)$ and the $K^{-}$multi-nucleon clusters.

The long-standing problem of the optical potential depth of the $K^{-}$-nucleus interaction could be solved by the high-resolution kaonic helium X-ray measurement with superconducting microcalorimeters. The J-PARC E62 group plans the measurement of both the $K^{-}{ }_{-}^{4} \mathrm{He}$ and $K^{-}{ }_{-}{ }^{3} \mathrm{He}$ $\mathrm{X}$-rays with a 240-pixel TES array. The difference of $2 p$-state strong-interaction shifts of $K^{-}{ }^{4} \mathrm{He}$ and $K^{-}{ }^{3} \mathrm{He}$ will be determined with the precision of $0.2 \mathrm{eV}$. The precise result can give a decisive settlement of the deep-or-shallow problem. This will be an important complement of the experimental direct search for the $K^{-}$-bound states in nuclei.

Acknowledgments We thank C. Capoccia, G. Corradi, B. Dulach, and D. Tagnani from LNF-INFN; and H. Schneider, L. Stohwasser, and D. Stuükler from Stefan-Meyer-Institut, for their fundamental contribution in designing and building the SIDDHARTA setup. We thank as well the DAФNE staff for the excellent working conditions and permanent support. Part of this work was supported by the European Community-Research Infrastructure Integrating Activity "Study of Strongly Interacting Matter" (Hadron-Physics2, Grant Agreement No. 227431, and Hadron-Physics3 (HP3) Contract No. 283286) under the Seventh Framework Programme of EU; Hadron-Physics I3 FP6 European Community program, Contract No. RII3-CT-2004-506078; Austrian Science Fund (FWF) (P24756-N20); Austrian Federal Ministry of Science and Research BMBWK 650962/0001 VI/2/2009; Romanian National Authority for Scientific Research, Contract No. 2-CeX 06-11-11/2006; the Grantin-Aid for Specially Promoted Research (20002003), MEXT, Japan; and the Croatian Science Foundation, under project HRZZ 1680.

\section{References}

[1] S. Deser, M.L. Goldberger, K. Baumann, W. Thirring, Phys. Rev. 96, 774 (1954); U.-G. Meißner, U. Raha, A. Rusetsky, Eur. Phys. J. C 35, 349 (2004)

[2] A. Gal, Nucl. Phys. A 914, 270 (2013)

[3] A. Cieplý, M. Mai, U.-G. Meißner, J. Smejkal, Nucl. Phys. A 954, 17 (2016)

[4] Y. Akaishi, T. Yamazaki, Phys. Rev. C 65, 044005 (2002)

[5] C.J. Batty, E. Friedman, A. Gal, Phys. Rep. 287, 385 (1997)

[6] E. Friedman, Int. J. Mod. Phys. A 26, 468 (2011)

[7] S. Hirenzaki, Y. Okumura, H. Toki, E. Oset, A. Ramos, Phys. Rev. C 61, 055205 (2000)

[8] M. Iwasaki et al., Phys. Rev. Lett. 78, 3097 (1997); T. M. Ito et al., Phys. Rev. C 58, 2366 (1998)

[9] G. Beer et al., Phys. Rev. Lett. 94, 212302 (2005)

[10] SIDDHARTA Collaboration, M. Bazzi, et al., Phys. Lett. B 704, 113 (2011)

[11] SIDDHARTA-2 Collaboration, Proposal of Laboratori Nazionali di Frascati of INFN, The upgrade of the SIDDHARTA apparatus for an enriched scientific case, 2010 
[12] J. Zmeskal et al., Proposal for J-PARC 30-GeV Proton Synchrotron, Measurement of the strong interaction induced shift and width of the 1st state of kaonic deuterium at J-PARC (J-PARC E57), 2014

[13] R.S. Hayano et al., Proposal for J-PARC 30-GeV Proton Synchrotron, Precision spectroscopy of kaonic atom X-rays with TES (J-PARC E62), 2015

[14] S. Okada, et al., Phys. Lett. B 653, 387 (2007)

[15] B. Borasoy, R. Nißler, W. Weise, Phys. Rev. Lett. 94, 213401 (2005); B. Borasoy, U.-G. Meißner, R. Nißler, Phys. Rev. C 74, 055201 (2006)

[16] SIDDHARTA Collaboration, M. Bazzi, et al., Nucl. Phys. A 954, 7 (2016)

[17] SIDDHARTA Collaboration, M. Bazzi, et al., Nucl. Phys. A 907, 69 (2013)

[18] M. Döring, U.-G. Meißner, Phys. Lett. B 704, 663 (2011); T. Mizutani, C. Fayard, B. Saghai, K. Tsushima, Phys. Rev. C 87, 035201 (2013); N.V. Shevchenko, Nucl. Phys. A 890-891, 50 (2012)

[19] SIDDHARTA Collaboration, M. Bazzi, et al., Phys. Lett. B 681, 310 (2009)

[20] SIDDHARTA Collaboration, M. Bazzi, et al., Phys. Lett. B 697, 199 (2011)

[21] SIDDHARTA Collaboration, M. Bazzi, et al., Phys. Lett. B 714, 40 (2012)

[22] H. Tatsuno, Precision X-ray spectroscopy of kaonic helium atom, Ph.D. Thesis, The University of Tokyo, 2011

[23] J. Yamagata-Sekihara, S. Hirenzaki, E. Hiyama et al. (private communication)

[24] J. Mareš, E. Friedman, A. Gal, Nucl. Phys. A 770, 84 (2006)

[25] A. Ramos, E. Oset, Nucl. Phys. A 671, 481 (2000)

[26] H. Tatsuno et al., J. Low. Temp. Phys. 184, 930 (2016)

[27] S. Okada et al., Prog. Theor. Exp. Phys., accepted; S. Okada et al., arXiv:1608.05436 